\title{
Existence and uniqueness of entropy solutions for nonlinear elliptic equations with measure data
}

by

\author{
Lucio BOCCARDO \\ Dipartimento di Matematica, Università di Roma I \\ P.le A. Moro 2, 00185, Roma, Italy \\ Thierry GALLOUËT \\ ENS-Lyon \\ 69364 Lyon Cedex 7, France \\ and \\ Luigi ORSINA \\ Dipartimento di Matematica, Università di Roma I \\ P.le A. Moro 2, 00185, Roma, Italy
}

ABSTRACT. - We consider the differential problem

$$
\left\{\begin{array}{cl}
A(u)=\mu & \text { in } \Omega \\
u=0 & \text { on } \partial \Omega
\end{array}\right.
$$

where $\Omega$ is a bounded, open subset of $\mathbf{R}^{N}, N \geq 2, A$ is a monotone operator acting on $W_{0}^{1, p}(\Omega), p>1$, and $\mu$ is a Radon measure on $\Omega$ that does not charge the sets of zero $p$-capacity. We prove a decomposition theorem for these measures (more precisely, as the sum of a function in $L^{1}(\Omega)$ and of a measure in $W^{-1, p^{\prime}}(\Omega)$ ), and an existence and uniqueness result for the so-called entropy solutions of $(*)$.

Key words: Representation of measures, uniqueness of entropy solutions.

AMS Classification: 35A05, 35R05.

Annales de l'Institut Henri Poincaré - Analyse non linéaire - 0294-1449

Vol. 13/96/05/\$ 4.00/

(C) 1996 L'Association Publications de l'Institut Henri Poincaré. Published by Elsevier B.V. All rights reserved 
RÉSUMÉ. - On considère le problème différentiel

$$
\left\{\begin{array}{cc}
A(u)=\mu & \text { dans } \Omega, \\
u=0 & \text { sur } \partial \Omega,
\end{array}\right.
$$

où $\Omega$ est un ouvert borné de $\mathbf{R}^{N}, N \geq 2, A$ est un opérateur monotone défini sur $W_{0}^{1, p}(\Omega), p>1$, et $\mu$ est une mesure de Radon sur $\Omega$ qui ne charge pas les ensembles de $p$-capacité nulle. On démontre un théorème de décomposition pour ces mesures (plus précisément, comme une fonction de $L^{1}(\Omega)$ et une mesure dans $\left.W^{-1, p^{\prime}}(\Omega)\right)$, et on prouve l'existence et l'unicité d'une solution entropique pour $(*)$.

\section{INTRODUCTION AND HYPOTHESES}

Let us consider the following nonlinear elliptic problem

$$
\left\{\begin{array}{cl}
A(u)=\mu & \text { in } \Omega, \\
u=0 & \text { on } \partial \Omega,
\end{array}\right.
$$

where $\Omega$ is a bounded open set in $\mathbf{R}^{N}, A$ is a nonlinear elliptic monotone operator in divergence form acting on $W_{0}^{1, p}(\Omega)(1<p<\infty)$, and $\mu$ is a Radon measure on $\Omega$ (as usual, we identify the measure $\mu$ with the application $f \rightarrow \int_{\Omega} f d \mu$ defined on $C(\bar{\Omega})$ ).

Existence of solutions for problem (1) if $A$ is a linear elliptic operator have been obtained, using duality techniques, by G. Stampacchia in [14]. As a consequence of the existence proof, there is also uniqueness of solutions. Unfortunately, the duality method only works in a linear setting, so that it was necessary to look for different techniques in order to deal with nonlinear problems. In the case of a datum $\mu$ in $L^{1}(\Omega)$, the first results were given by $\mathrm{H}$. Brezis and W. Strauss (see [5]), where more general equations (with maximal monotone graphs) were studied. See also [4]. Subsequently, existence results for the general case ( $\mu$ a Radon measure, and a monotone operator $A$ ) were proved in [2] and [3]. However, only existence results were given, the uniqueness problem being a rather delicate one, due to a counterexample by J. Serrin (see [13], and [12] for further remarks). The first results towards uniqueness were given by A. Dall'Aglio in [6], where it was proved for strongly monotone operators and $L^{1}(\Omega)$ data; the result was, however, not "intrinsic", since there was only uniqueness of solutions obtained by an approximation technique. 
In a recent paper (see [1]) has been introduced a new notion of solution for (1) if $\mu$ is an $L^{1}$ function, with the precise purpose of proving its uniqueness: the so-called entropy solution. In that paper it was proved both the existence and the uniqueness of an entropy solution. However, the method of [1] is confined to the case of an $L^{1}$ datum, since, in particular, the concept of entropy solution is meaningless if the datum is a Radon measure. In this paper we extend the result of [1], taking into account a signed measure $\mu$ which is zero on the subsets of zero $p$-capacity (i.e., the capacity defined starting from $W_{0}^{1, p}(\Omega)$ ). In order to do this, we prove a decomposition theorem for these measures: every signed measure that is zero on the sets of zero $p$-capacity can be splitted in the sum of an element in $W^{-1, p^{\prime}}(\Omega)$ (the dual space of $W_{0}^{1, p}(\Omega)$ ), and of a function in $L^{1}(\Omega)$, and, conversely, every signed measure in $L^{1}(\Omega)+W^{-1, p^{\prime}}(\Omega)$ is zero on the sets of zero $p$-capacity. One of the tools of the proof will be a result of G. Dal Maso (see [7]), which states that these measures can be decomposed as the product of a Borel function and of a measure in $W^{-1, p^{\prime}}(\Omega)$.

Using the decomposition of measures, we prove, following the lines of [1], that there exists a unique entropy solution of (1). This proof will strongly rely on the structure of the measure $\mu$, that is, $\mu$ belongs to $L^{1}(\Omega)+W^{-1, p^{\prime}}(\Omega)$. To better specify our result, we will also provide an example (in the linear case) showing how the definition of entropy solution is not suitable in order to have uniqueness if the measure $\mu$ is the Dirac mass.

We begin stating the hypotheses that will hold throughout the paper.

Let $\Omega$ be a bounded, open subset of $\mathbf{R}^{N}, N \geq 2$. Let $p$ be a real number such that $1<p \leq N$. Observe that, for the purposes of this paper, the case $p>N$ can be omitted, since the set of all signed measures on $\Omega$ is a subset of $W^{-1, p^{\prime}}(\Omega)$, so that the existence and uniqueness results are a consequence of the variational theory.

Let $a: \Omega \times \mathbf{R}^{N} \rightarrow \mathbf{R}^{N}$ be a Carathéodory function such that the following holds:

$$
a(x, \xi) \cdot \xi \geq \alpha|\xi|^{p},
$$

for almost every $x \in \Omega$, for every $\xi \in \mathbf{R}^{N}$, where $\alpha$ is a positive constant;

$$
|a(x, \xi)| \leq \lambda(x)+\beta|\xi|^{p-1},
$$

for almost every $x \in \Omega$, for every $\xi \in \mathbf{R}^{N}$, where $\beta$ is a positive constant, and $\lambda$ belongs to $L^{p^{\prime}}(\Omega)$;

$$
(a(x, \xi)-a(x, \eta)) \cdot(\xi-\eta)>0,
$$

Vol. 13, $\mathrm{n}^{\circ}$ 5-1996. 
for almost every $x \in \Omega$, for every $\xi$ and $\eta$ in $\mathbf{R}^{N}$, with $\xi \neq \eta$.

Let us define the differential operator

$$
A(u)=-\operatorname{div}(a(x, \nabla u)) .
$$

Thanks to (2), (3) and (4), $A$ is a monotone and coercive differential operator acting between $W_{0}^{1, p}(\Omega)$ and $W^{-1, p^{\prime}}(\Omega)$; hence, it is surjective (see [11]).

Let $K$ be a compact subset of $\Omega$. The $p$-capacity of $K$ with respect to $\Omega$ is defined as:

$$
\operatorname{cap}_{p}(K, \Omega)=\inf \left\{\int_{\Omega}|\nabla u|^{p} d x: u \in C_{0}^{\infty}(\Omega), u \geq \chi_{K}\right\},
$$

where $\chi_{K}$ is the characteristic function of $K$; we use the convention that $\inf \emptyset=+\infty$. This definition can be extended to any open subset $B$ of $\Omega$ in the following way:

$$
\operatorname{cap}_{p}(B, \Omega)=\sup \left\{\operatorname{cap}_{p}(K, \Omega), K \text { compact, } K \subset B\right\} .
$$

Finally, it is possible to define the $p$-capacity of any borelian set $A$ as

$$
\operatorname{cap}_{p}(A, \Omega)=\inf \left\{\operatorname{cap}_{p}(B, \Omega), B \text { open, } A \subset B\right\} \text {. }
$$

We also remark that, once the $p$-capacity has been defined as before, then, for every subset $B$ of $\Omega$,

$$
\operatorname{cap}_{p}(B, \Omega)=\inf \left\{\int_{\Omega}|\nabla u|^{p} d x\right\},
$$

and the infimum is taken on all functions $u \in W_{0}^{1, p}(\Omega)$ such that $u=1$ $\operatorname{cap}_{p}$-quasi everywhere on $B, u \geq 0$ cap $_{p}$-quasi everywhere on $\Omega$. In the preceding assertion we have taken for $u$ its $\operatorname{cap}_{p}$-quasi continuous representative.

We will denote by $\mathcal{M}_{\mathrm{b}}(\Omega)$ the space of all signed measures on $\Omega$, i.e., the space of all $\sigma$-additive set functions $\mu$ with values in $\mathbf{R}$ defined on the Borel $\sigma$-algebra. Note that, if $\mu$ belongs to $\mathcal{M}_{\mathrm{b}}(\Omega)$, then $|\mu|$ (the total variation of $\mu$ ) is a bounded positive measure on $\Omega$. We will denote by $\mathcal{M}_{\mathrm{b}}^{p}(\Omega)$ the space of all measures $\mu$ in $\mathcal{M}_{\mathrm{b}}(\Omega)$ such that $\mu(E)=0$ for every set such that $\operatorname{cap}_{p}(E, \Omega)=0$.

We define, for $s$ and $k$ in $\mathbf{R}$, with $k \geq 0, T_{k}(s)=\max (-k, \min (k, s))$.

Let $p$ be a real number, with $1<p<+\infty$. Following [1], we define $\mathcal{T}_{0}^{1, p}(\Omega)$ as the set of (classes of) measurable functions $u$ on $\Omega$ such that $T_{k}(u)$ belongs to $W_{0}^{1, p}(\Omega)$ for every $k>0$.

The outline of the paper is as follows: in the next section we will prove that a signed measure $\mu$ belongs to $\mathcal{M}_{\mathrm{b}}^{p}(\Omega)$ if and only if it is the sum of an element of $W^{-1, p^{\prime}}(\Omega)$ and of a function in $L^{1}(\Omega)$. The third section will study existence and uniqueness of entropy solutions for (1). 


\section{DECOMPOSITION OF A MEASURE IN $\mathcal{M}_{\mathrm{b}}^{p}(\Omega)$}

We are going to prove the following result

THEOREM 2.1. - Let $p$ be a real number such that $1<p<+\infty$. Let $\mu$ be an element of $\mathcal{M}_{\mathrm{b}}(\Omega)$. Then $\mu \in L^{1}(\Omega)+W^{-1, p^{\prime}}(\Omega)$ if and only if $\mu \in \mathcal{M}_{\mathrm{b}}^{p}(\Omega)$.

Proof. - Let $\mu$ be a measure in $\mathcal{M}_{\mathrm{b}}(\Omega)$.

If $\mu$ belongs to $L^{1}(\Omega)+W^{-1, p^{\prime}}(\Omega)$ then it is in $\mathcal{M}_{\mathrm{b}}^{p}(\Omega)$. This is the easy part of the proof, and we leave it to the reader.

Hence, we only need to prove the converse, that is, if $\mu$ is in $\mathcal{M}_{\mathrm{b}}^{p}(\Omega)$, then $\mu$ belongs to $L^{1}(\Omega)+W^{-1, p^{\prime}}(\Omega)$.

Step 1. - We first observe that if $\operatorname{cap}_{p}(A, \Omega)=0$, then both $\mu^{+}(A)$ and $\mu^{-}(A)$ are zero. This is an obvious consequence of the definition of $\mu^{+}$and $\mu^{-}$, and of the monotonicity of $p$-capacity. Indeed, $\mu^{+}(A)=$ $\sup \{\mu(B): B$ borelian, $B \subset A\}$. Therefore, $\mu$ may be assumed to be positive in the sequel.

Step 2. - By the result of [7], every positive measure $\mu$ in $\mathcal{M}_{\mathrm{b}}^{p}(\Omega)$ can be decomposed as $\mu=h \gamma$, with $h$ a positive Borel measurable function in $L^{1}(\Omega, \gamma)$ and $\gamma$ a positive measure in $W^{-1, p^{\prime}}(\Omega)$. Let $\left\{K_{n}\right\}$ be an increasing sequence of compact sets contained in $\Omega$ such that $\bigcup_{n=1}^{+\infty} K_{n}=\Omega$, and let $\mu_{n}^{(1)}=T_{n}\left(h \chi_{K_{n}}\right) \gamma$. It is easy to see that $\left\{\mu_{n}^{(1)}\right\}$ is an increasing sequence of positive measures in $W^{-1, p^{\prime}}(\Omega)$ with compact support in $\Omega$. Let $\mu_{0}=\mu_{0}^{(1)}$ and $\mu_{n}=\mu_{n}^{(1)}-\mu_{n-1}^{(1)}$. Then $\mu=\sum_{n=1}^{+\infty} \mu_{n}$, and the series converges strongly in $\mathcal{M}_{\mathrm{b}}(\Omega)$. In particular, $\sum_{n=1}^{+\infty}\left\|\mu_{n}\right\|_{\mathcal{M}_{\mathrm{b}}(\Omega)}<\infty$ (note that $\left\|\mu_{n}\right\|_{\mathcal{M}_{\mathrm{b}}(\Omega)}=\mu_{n}(\Omega)$ since $\left.\mu_{n} \geq 0\right)$.

Step 3. - Let $\rho$ be a function in $C_{0}^{\infty}\left(\mathbf{R}^{N}\right)$, such that $\rho(x) \geq 0$ everywhere, and $\int_{\mathbf{R}^{N}} \rho(x) d x=1$. Let $\left\{\rho_{n}\right\}$ be the sequence of mollifiers associated to $\rho$, i.e., $\rho_{n}(x)=n^{N} \rho(n x)$ for every $x \in \mathbf{R}^{N}$. For $n \in \mathbf{N}$, if $\mu_{n}$ is the measure defined in Step 2, we have that $\left\{\mu_{n} * \rho_{m}\right\}$ converges to $\mu_{n}$ in $W^{-1, p^{\prime}}(\Omega)$ as $m$ tends to infinity. By the properties of $\mu_{n}$ and $\rho_{m}, \mu_{n} * \rho_{m}$ belongs to $C_{0}^{\infty}(\Omega)$ if $m$ is large enough.

Choose $m=m_{n}$ such that $\mu_{n} * \rho_{m_{n}}$ belongs to $C_{0}^{\infty}(\Omega)$ and $\left\|\mu_{n} * \rho_{m_{n}}-\mu_{n}\right\|_{W^{-1, p^{\prime}}(\Omega)} \leq 2^{-n}$. Then $\mu_{n}=f_{n}+g_{n}$, where $f_{n}=\mu_{n} * \rho_{m_{n}}$ and $g_{n}=\mu_{n}-\mu_{n} * \rho_{m_{n}}$. Thanks to the choice of $m_{n}$, the series $\sum_{n=1}^{+\infty} g_{n}$ converges in $W^{-1, p^{\prime}}(\Omega)$, and so $g=\sum_{n=1}^{+\infty} g_{n}$ belongs to $W^{-1, p^{\prime}}(\Omega)$. Since $\left\|f_{n}\right\|_{L^{1}(\Omega)}=\left\|\mu_{n} * \rho_{m_{n}}\right\|_{L^{1}(\Omega)} \leq\left\|\mu_{n}\right\|_{\mathcal{M}_{\mathrm{b}}(\Omega)}$, by Step 2 the series $\sum_{n=1}^{+\infty} f_{n}$ 
is absolutely convergent in $L^{1}(\Omega)$, and so $f=\sum_{n=1}^{+\infty} f_{n}$ belongs to $L^{1}(\Omega)$. Thus, the three series $\sum_{n=1}^{+\infty} \mu_{n}, \sum_{n=1}^{+\infty} g_{n}$, and $\sum_{n=1}^{+\infty} f_{n}$ converge in the sense of distributions, and so $\mu=f+g$. This completes the proof.

Remark 2.2. - The previous proof remains the same also for measures that are zero on the sets of zero $(k, p)$-capacity (i.e., the capacity defined starting from $W_{0}^{k, p}(\Omega), k>1$ ), since the result of [7] states that these measures can be decomposed as $h \gamma$, with $h$ a Borel function and $\gamma$ a measure of $W^{-k, p^{\prime}}(\Omega)$. Thus, it is possible to prove that every signed measure on $\Omega$ which is zero on the sets of zero $(k, p)$-capacity can be decomposed in the sum of an element in $W^{-k, p^{\prime}}(\Omega)$ and of a function in $L^{1}(\Omega)$, and vice versa. This result has been proved, for $k=2$, by Gallouët and Morel in [10]; since we are interested in second order elliptic equations, we have preferred to restrict our attention to $\mathcal{M}_{\mathrm{b}}^{p}(\Omega)$ (i.e., to $L^{1}(\Omega)+W^{-1, p^{\prime}}(\Omega)$ ).

Remark 2.3. - From the proof of the theorem, it is clear that if $\mu$ is a positive measure, then $f$ (the $L^{1}(\Omega)$ part) is positive. This is not true in general for $g$ (the $W^{-1, p^{\prime}}(\Omega)$ part). Actually, let $\mu=\mu_{a}+\mu_{s}$ be the Lebesgue decomposition of $\mu$, with $\mu_{a} \in L^{1}(\Omega)$ and $\mu_{s}$ singular with respect to the Lebesgue measure in $\mathbf{R}^{N}$. Then there exists a positive $g$ in $W^{-1, p^{\prime}}(\Omega)$ such that $\mu-g$ belongs to $L^{1}(\Omega)$ if and only if $\mu_{s}$ belongs to $W^{-1, p^{\prime}}(\Omega)$. If $\mu_{s}$ is in $W^{-1, p^{\prime}}(\Omega)$, we can choose $g=\mu_{s}$. On the other hand, if there exists such a $g$, then $\mu_{s}=g_{s}$; moreover, since $g$ and $g_{s}$ are positive, then $0 \leq g_{a} \leq g$, and so $g_{a}$ belongs to $W^{-1, p^{\prime}}(\Omega)$; hence, $g_{s}=g-g_{a} \in W^{-1, \overline{p^{\prime}}}(\Omega)$ (this has already been noted ([8])).

To complete this section, let us recall the following result.

Theorem 2.4. - Let $\mu$ be a positive Radon measure on $\Omega$. Then there exists a unique pair $\left(\mu_{0}, \mu_{1}\right)$ of Radon measures on $\Omega$ such that

1) $\mu=\mu_{0}+\mu_{1}$

2) $\mu_{0} \in \mathcal{M}_{\mathrm{b}}^{p}(\Omega)$

3) $\mu_{1}(E)=\mu(E \cap N)$, for some Borel set $N$ such that $\operatorname{cap}_{p}(N, \Omega)=0$, for every $\mu$-measurable set $E$ in $\Omega$.

Proof. - See [9], Lemma 2.1.

Thus, in view of Theorem 2.1 , every positive Radon measure $\mu$ on $\Omega$ can be written as

$$
\mu=\mu_{0}+\mu_{1}+\mu_{2}
$$

with $\mu_{0} \in W^{-1, p^{\prime}}(\Omega), \mu_{1} \in L^{1}(\Omega), \mu_{1} \geq 0, \mu_{2}(E)=\mu(E \cap N)$, with $\operatorname{cap}_{p}(N, \Omega)=0$. 


\section{ENTROPY SOLUTIONS: EXISTENCE AND UNIQUENESS}

We begin by giving the definition of entropy solution for (1), following [1].

Definition 3.1. - Let $\mu$ be a signed measure in $L^{1}(\Omega)+W^{-1, p^{\prime}}(\Omega)$ (that is, $\mu$ belongs to $\mathcal{M}_{\mathrm{b}}^{p}(\Omega)$ ). A function $u$ in $\mathcal{T}_{0}^{1, p}(\Omega)$ is an entropy solution of the equation

$$
\left\{\begin{array}{cl}
A(u)=\mu & \text { in } \Omega \\
u=0 & \text { on } \partial \Omega
\end{array}\right.
$$

if

$$
\int_{\Omega} a(x, \nabla u) \cdot \nabla T_{k}(u-\phi) d x \leq \int_{\Omega} T_{k}(u-\phi) d \mu,
$$

for every $\phi$ in $\mathcal{D}^{\prime}(\Omega)$ and for every $k>0$. If $p<2-\frac{1}{N}$ then the derivative $\nabla u$ is not the derivative in distribution sense, but is defined (we recall that $u$ belongs to $\mathcal{T}_{0}^{1, p}(\Omega)$ ) as $\nabla u=\nabla T_{k}(u)$ on the set $\{|u|<k\}$. We remark explicitly that the right hand side of (6) is well defined, since $T_{k}(u-\phi)$ belongs to $W_{0}^{1, p}(\Omega) \cap L^{\infty}(\Omega)$.

It is possible to show, reasoning as in [1], Lemma 3.3, that if $u$ is an entropy solution, then (6) holds for every function $\phi$ in $\mathcal{T}_{0}^{1, p}(\Omega) \cap L^{\infty}(\Omega)$. In particular, it holds if $\phi=T_{k}(v)$, with $v$ in $\mathcal{T}_{0}^{1, p}(\Omega)$. Thus, choosing $\phi=T_{h}(u)$, and writing $\mu=f-\operatorname{div}(F)$, with $f \in L^{1}(\Omega)$ and $F \in\left(L^{p^{\prime}}(\Omega)\right)^{N}$, we have

$$
\begin{aligned}
\int_{\Omega} a(x, \nabla u) \cdot \nabla T_{k}\left(u-T_{h}(u)\right) d x & \leq \int_{\Omega} f T_{k}\left(u-T_{h}(u)\right) d x \\
& +\int_{\Omega} F \cdot \nabla T_{k}\left(u-T_{h}(u)\right) d x .
\end{aligned}
$$

Since $\nabla T_{k}\left(u-T_{h}(u)\right)=\nabla u$ where $h \leq|u| \leq h+k$, and is zero elsewhere, we can write

$$
\int_{\{h \leq|u| \leq h+k\}} a(x, \nabla u) \cdot \nabla u d x \leq k \int_{\{|u| \geq h\}}|f| d x+\int_{\{h \leq|u| \leq h+k\}} F \cdot \nabla u d x .
$$

Using (2) (in the left hand side), and Young inequality (in the right hand side), we get, setting $B_{h, k}=\{h \leq|u| \leq h+k\}$ and $A_{h}=\{|u| \geq h\}$,

$$
\alpha \int_{B_{h, k}}|\nabla u|^{p} d x \leq k \int_{A_{h}}|f| d x+c \int_{B_{h, k}}|F|^{p^{\prime}} d x+\frac{\alpha}{2} \int_{B_{h, k}}|\nabla u|^{p} d x
$$


Hence, if $u$ is an entropy solution of (5) we have, for every positive real numbers $h$ and $k$,

$$
\int_{B_{h, k}}|\nabla u|^{p} d x \leq c k \int_{A_{h}}|f| d x+c \int_{B_{h, k}}|F|^{p^{\prime}} d x
$$

so that, in particular, if $k$ is a fixed positive real number,

$$
\lim _{h \rightarrow \infty} \int_{B_{h, k}}|\nabla u|^{p} d x=0
$$

The following theorem can be proved exactly as in [1].

THEOREM 3.2. - Let $\mu$ be a signed measure in $L^{1}(\Omega)+W^{-1, p^{\prime}}(\Omega)$. Then there exists at least one entropy solution $u$ in $\mathcal{T}_{0}^{1, p}(\Omega)$ of $(5)$.

Every entropy solution $u$ is also a solution in the distribution sense; actually it satisfies

$$
\int_{\Omega} a(x, \nabla u) \cdot \nabla v d x=\int_{\Omega} v d \mu \quad \forall v \in C_{0}^{\infty}(\Omega) .
$$

Moreover, if $p>2-\frac{1}{N}$, then $u$ belongs to $W_{0}^{1, q}(\Omega)$, for every $q<\frac{N(p-1)}{N-1}$. In the case of a general $p$, then $|\nabla u|$ belongs to $M^{\frac{N(p-1)}{N-1}}(\Omega)$, the Marcinkiewicz space (note that, if $p \leq 2-\frac{1}{N}$, then $\frac{N(p-1)}{N-1} \leq 1$ ).

We are now ready to prove the uniqueness result for entropy solutions.

THEOREM 3.3. - Let $\mu$ be a signed measure in $L^{1}(\Omega)+W^{-1, p^{\prime}}(\Omega)$. Then the entropy solution is unique.

Proof. - By the hypotheses, $\mu$ can be written as $f-\operatorname{div}(F)$, with $f$ in $L^{1}(\Omega)$, and $F \in\left(L^{p^{\prime}}(\Omega)\right)^{N}$. Suppose that $u$ and $v$ are two entropy solutions relative to $\mu$; choose $T_{k}\left(u-T_{h}(v)\right)$ as test function in (6) (written for $u$ ) and $T_{k}\left(v-T_{h}(u)\right)$ as test function in (6) (written for $v$ ). Add the equations, so that

$$
\begin{aligned}
\int_{\Omega} & {[a(x, \nabla u)-F] \cdot \nabla T_{k}\left(u-T_{h}(v)\right) } \\
& +\int_{\Omega}[a(x, \nabla v)-F] \cdot \nabla T_{k}\left(v-T_{h}(u)\right) \\
\leq & \int_{\Omega} f\left[T_{k}\left(u-T_{h}(v)\right)+T_{k}\left(v-T_{h}(u)\right)\right] .
\end{aligned}
$$


The right hand side of the preceding relation tends to zero as $h$ tends to infinity, since $T_{k}(s)$ is odd. For the left hand side, let us define (we have omitted the dependence on $x \in \Omega$ for the sake of brevity)

$$
\begin{gathered}
C_{0}=\{|u-v| \leq k,|u| \leq h,|v| \leq h\}, \\
C_{1}=\left\{\left|u-T_{h}(v)\right| \leq k,|v|>h\right\}, \quad C_{1}^{\prime}=\left\{\left|v-T_{h}(u)\right| \leq k,|u|>h\right\}, \\
C_{2}=\left\{\left|u-T_{h}(v)\right| \leq k,|v| \leq h,|u|>h\right\}, \\
C_{2}^{\prime}=\left\{\left|v-T_{h}(u)\right| \leq k,|u| \leq h,|v|>h\right\},
\end{gathered}
$$

so that $\Omega=C_{0} \cup C_{1} \cup C_{2}=C_{0} \cup C_{1}^{\prime} \cup C_{2}^{\prime}$. On $C_{0}$ the left hand side is equal to

$$
\int_{C_{0}}[a(x, \nabla u)-a(x, \nabla v)] \cdot \nabla(u-v) d x .
$$

On $C_{1}$ (and on $C_{1}^{\prime}$ with $u$ exchanged with $v$ ), recalling (2), we have

$$
\int_{C_{1}}[a(x, \nabla u)-F] \cdot \nabla u d x \geq-\int_{C_{1}} F \cdot \nabla u d x .
$$

By Hölder inequality,

$$
\int_{C_{1}} F \cdot \nabla u d x \leq\left(\int_{C_{1}}|F|^{p^{\prime}} d x\right)^{\frac{1}{p^{\prime}}}\left(\int_{C_{1}}|\nabla u|^{p} d x\right)^{\frac{1}{p}} .
$$

Since $\left|C_{1}\right|$ tends to zero as $h$ tends to infinity, we have that $\int_{C_{1}}|F|^{p^{\prime}} d x$ tends to zero; if we prove that $\int_{C_{1}}|\nabla u|^{p} d x$ is bounded with respect to $h$, then the term with $C_{1}$ (and so with $C_{1}^{\prime}$ ) will converge to zero. We decompose $C_{1}$ as

$$
C_{1}=\{v>h,|u-h| \leq k\} \cup\{v<-h,|u+h| \leq k\}=C_{1}^{+} \cup C_{1}^{-} .
$$

On $C_{1}^{+}$(and the same holds for $C_{1}^{-}$) we have $-k \leq u-h \leq k$, and so $h-k \leq u \leq h+k$. Hence $C_{1}^{+} \subseteq B_{h-k, 2 k}$. Thus, by (7),

$$
\int_{C_{1}}|\nabla u|^{p} d x \leq \int_{B_{h-k, 2 k}}|\nabla u|^{p} d x \leq c,
$$

that is what we wanted to prove; hence,

$\limsup _{h \rightarrow \infty}\left(\int_{C_{1}}[a(x, \nabla u)-F] \cdot \nabla u d x+\int_{C_{1}^{\prime}}[a(x, \nabla v)-F] \cdot \nabla v d x\right) \geq 0$. 
On $C_{2}$ (and the same estimates can be done on $C_{2}^{\prime}$ ), we have, by (2),

$$
\begin{aligned}
\int_{C_{2}}[a(x, \nabla u)-F] \cdot \nabla(u-v) d x \geq & -\int_{C_{2}}[a(x, \nabla u)-F] \cdot \nabla v d x \\
& -\int_{C_{2}} F \cdot \nabla u d x .
\end{aligned}
$$

Reasoning as before, the second term tends to zero as $h$ tends to infinity if $\int_{C_{2}}|\nabla u|^{p} d x$ is bounded by a constant independent on $h$, since $\left|C_{2}\right|$ tends to zero as $h$ diverges. We split $C_{2}$ as $C_{2}^{+} \cup C_{2}^{-}$, where

$C_{2}^{+}=\{|u-v| \leq k,|v| \leq h, u>h\}, C_{2}^{-}=\{|u-v| \leq k,|v| \leq h, u<-h\}$.

On $C_{2}^{+}$(and $C_{2}^{-}$can be treated in the same way), we have $v \leq h$ and $-k \leq u-v \leq k$, so that $v-k \leq u \leq v+k \leq h+k$; since $v-k<h$, then $C_{2}^{+} \subseteq B_{h, k}$. Using (7) again, we obtain the result. For the first term we have, as in [1],

$$
\begin{aligned}
\int_{C_{2}}[a(x, \nabla u)-F] \cdot \nabla v \leq & \left(\int_{\{h \leq|u| \leq h+k\}}\left[|\nabla u|^{p-1}+|F|\right]^{p^{\prime}}\right)^{\frac{1}{p^{\prime}}} \\
& \times\left(\int_{\{h-k \leq|v| \leq h\}}|\nabla v|^{p}\right)^{\frac{1}{p}},
\end{aligned}
$$

and the right hand side tends to zero as $h$ tends to infinity. Summing up the results obtained for $C_{0}, C_{1}, C_{1}^{\prime}, C_{2}$ and $C_{2}^{\prime}$, we have

$$
\lim _{h \rightarrow \infty} \int_{C_{0}}[a(x, \nabla u)-a(x, \nabla v)] \cdot \nabla(u-v) d x=0
$$

that is,

$$
\int_{\{|u-v| \leq k\}}[a(x, \nabla u)-a(x, \nabla v)] \cdot \nabla(u-v) d x=0,
$$

for every $k>0$. Thus, by (4), $\nabla u=\nabla v$ almost everywhere, and so $u=v$.

Remark 3.4. - We are going to prove that if $\mu$ charges the sets of $p$ capacity zero, then the notion of entropy solution is not suitable in order to obtain uniqueness of solutions. Actually, let $N \geq 2, \Omega=B_{1}(0)$, and 
$\mu=\delta_{0}$, the Dirac mass concentrated in the origin of $\mathbf{R}^{N}$. Let us consider the following problem

$$
\left\{\begin{array}{cl}
-\Delta u=\delta_{0} & \text { in } \Omega \\
u=0 & \text { on } \partial \Omega
\end{array}\right.
$$

It is known that (8) has a unique solution $u$ in the sense of distributions belonging to $W_{0}^{1,1}(\Omega)$; it can be explicitly calculated, and is (we restrict our example to the case $N \geq 3$ for simplicity) $u(x)=c_{N}\left(|x|^{2-N}-1\right)$, with $c_{N}$ a positive constant depending only on the dimension $N$. We are going to prove that $\alpha u$ is an entropy solution of (8) (that is, it satisfies (9) below) for every real number $\alpha$ such that $0<\alpha \leq 1$. We begin by proving this fact for $\alpha=1$, that is

$$
\int_{\Omega} \nabla u \cdot \nabla T_{k}(u-\phi) d x \leq \int_{\Omega} T_{k}(u-\phi) d \delta_{0} \quad \forall \phi \in C_{0}^{\infty}(\Omega), \forall k>0
$$

Let $f_{n}=\chi_{B_{\frac{1}{n}}(0)} / \operatorname{meas}\left(B_{\frac{1}{n}}(0)\right)$. As it is well known, $f_{n}$ converges to $\delta_{0}$ in the weak-* topology of measures. Let $u_{n}$ be the solution of

$$
\left\{\begin{array}{cl}
-\Delta u_{n}=f_{n} & \text { in } \Omega \\
u_{n}=0 & \text { on } \partial \Omega
\end{array}\right.
$$

By the results of [2], $\left\{u_{n}\right\}$ converges to $u$ in $W_{0}^{1, q}(\Omega)$ for every $q<\frac{N}{N-1}$. On the other hand, it is easy to see (also $u_{n}$ can be explicitly calculated) that $u_{n}$ is greater than $c_{N}\left(|n|^{2-N}-1\right)$ on $B_{\frac{1}{n}}(0)$, so that, for fixed $k$ and $\phi$,

$$
\int_{\Omega} f_{n} T_{k}\left(u_{n}-\phi\right) d x=k=\int_{\Omega} T_{k}(u-\phi) d \delta_{0}
$$

(observe that the last expression has sense because $T_{k}(u-\phi)$ is continuous). Moreover, using the explicit expression of $u_{n}$, for every fixed $k>0$, there exists $n(k) \in \mathbf{N}$ such that $T_{k}\left(u_{n}\right)$ is equal to $T_{k}(u)$ for every $n>n(k)$. Thus, using the properties of $u_{n}$, and recalling that the test functions $\phi$ are bounded,

$$
\lim _{n \rightarrow \infty} \int_{\Omega} \nabla u_{n} \cdot \nabla T_{k}\left(u_{n}-\phi\right) d x=\int_{\Omega} \nabla u \cdot \nabla T_{k}(u-\phi) d x
$$

and so $u$ is an entropy solution of (8), in the sense that (9) holds with $\leq$ replaced by $=$. Note that this fact is true not only for $\delta_{0}$, but also for any other datum of the form $\delta_{a}, a \in \Omega$ (and for the corresponding usual 
weak solution of (8)). Let now $\alpha$ be a real number in $(0,1)$. Then, since $u$ is an entropy solution,

$$
\begin{aligned}
& \int_{\Omega} \nabla(\alpha u) \cdot \nabla T_{k}(\alpha u-\phi) d x \\
& \quad=\alpha^{2} \int_{\left\{\left|u-\frac{\phi}{\alpha}\right| \leq \frac{k}{\alpha}\right\}} \nabla u \cdot \nabla\left(u-\frac{\phi}{\alpha}\right) d x \leq \alpha^{2} \frac{k}{\alpha} \leq k
\end{aligned}
$$

and so $\alpha u$ is an entropy solution of (8); i.e., the entropy solution is not unique. Observe that $\alpha u$ is not a solution in the distribution sense of (8) if $\alpha \neq 1$.

One can think that there exists at most a unique function $u$ that satisfies (9) with $\leq$ replaced by $=$. This is not true. Actually, let $u$ as before, and let $v$ be the solution of equation (8) where $\delta_{0}$ is replaced by $\delta_{a}$, with $a \in \Omega$, $a \neq 0$. Then $u_{\theta}=\theta u+(1-\theta) v$ is a solution of (9) with $\leq$ replaced by $=$ for every $\theta \in(0,1)$. This gives infinitely many solutions.

However, if $\mu$ belongs to $\mathcal{M}_{\mathrm{b}}^{p}(\Omega)$, then the entropy solution is also a solution in the sense of distributions, as we said before. In other words, the behaviour of a solution $u$ around its blow-up points (behaviour that is not considered in the formulation of entropy solutions), turns out to be unimportant if $\mu$ does not charge the sets of zero $p$-capacity, but it has to be considered if this is not the case.

\section{ACKNOWLEDGEMENTS}

We would like to thank Haim Brezis, Gianni Dal Maso, Annalisa Malusa and François Murat for some stimulating discussions. The first and third author have been supported by the Italian Government MURST $40 \%$ and $60 \%$ research funds.

\section{REFERENCES}

[1] P. Benilan, L. Boccardo, T. Gallouët, R. Gariepy, M. Pierre and J. L. Vazquez, An $L^{1}$ theory of existence and uniqueness of nonlinear elliptic equations, Ann. Scuola Norm. Sup. Pisa, Vol. 22, n. 2, 1995, pp. 240-273.

[2] L. BocCARDo and T. GAllouËT, Nonlinear elliptic and parabolic equations involving measure data, J. Funct. Anal., Vol. 87, 1989, pp. 149-169.

[3] L. BocCARDo and T. GAllouËT, Nonlinear elliptic equations with right hand side measures, Comm. Partial Differential Equations, Vol. 17, n. 3\&4, 1992, pp. 641-655.

[4] H. BREZIS, Some variational problems of the Thomas-Fermi type, in Variational inequalities and complementarity problems, Cottle, Gianessi, and Lions eds., Wiley, New York, 1980, pp. 53-73. 
[5] H. Brezis and W. Strauss, Semi-linear second-order elliptic equations in $L^{1}, J$. Math. Soc. Japan, Vol. 25, n. 4, 1973, pp. 565-590.

[6] A. Dall'Aglio, Approximated solutions of equations with $L^{1}$ data. Application to the $H$-convergence of parabolic quasi-linear equations, Ann. Mat. Pura Appl., Vol. 170, 1996, pp. 207-240.

[7] G. DAL MASO, On the integral representation of certain local functionals, Ricerche Mat., Vol. 22, 1983, pp. 85-113.

[8] G. Dal Maso, Personal communication.

[9] M. Fukushima, K. Sato and S. Taniguchi, On the closable part of pre-Dirichlet forms and the fine supports of underlying measures, Osaka J. Math., Vol. 28, 1991, pp. 517-535.

[10] T. GallouËt and J. M. Morel, Resolution of a semilinear equation in $L^{1}$, Proc. Roy. Soc. Edinburgh, Vol. 96, 1984, pp. 275-288.

[11] J. LERAY and J. L. Lions, Quelques résultats de Višik sur les problèmes elliptiques semilinéaires par les méthodes de Minty et Browder, Bull. Soc. Math. France, Vol. 93, 1965, pp. 97-107.

[12] A. PRIGNET, Remarks on existence and uniqueness of solutions of elliptic problems with right-hand side measures, Rend. Mat., Vol. 15, 1995, pp. 321-337.

[13] J. SERRIN, Pathological solutions of elliptic differential equations, Ann. Scuola Norm. Sup. Pisa, Vol. 18, 1964, pp. 385-387.

[14] G. STAMPACCHIA, Le problème de Dirichlet pour les équations elliptiques du second ordre à coefficients discontinus, Ann. Inst. Fourier, (Grenoble), Vol. 15, n. 1, 1965, pp. 189-258.

(Manuscript received August 15, 1994;

revised version received April 20, 1995.) 